\title{
A statistical study of field-aligned electron beams associated with ion conics events
}

\author{
W. Miyake ${ }^{1}$, T. Mukai ${ }^{2}$, N. Kaya ${ }^{3}$ \\ ${ }^{1}$ Communications Research Laboratory, 4-2-1 Koganei, Tokyo 184, Japan Fax: + 8142327 6657; e-mail: miyake@crl go.jp \\ ${ }^{2}$ The Institute of Space and Astronautical Science, Yoshinodai, Sagamihara 229 , Japan \\ ${ }^{3}$ Kobe University, 1-1, Rokkodai, Nada, Kobe 657, Japan
}

Received: 13 November 1997 / Revised: 9 February 1998 / Accepted: 16 February 1998

\begin{abstract}
A statistical study of field-aligned electron beams associated with upflowing ion conics is presented from Exos-D (Akebono) observations below $10000 \mathrm{~km}$. The electron beams are narrowly collimated along the field line and generally have energies of several tens of $\mathrm{eV}$. They are divided in the analysis into three types: upflowing, downflowing, and counterstreaming. All the types of electron beams are almost equivalent in their energy and pitch angle characteristics and their association rate with upflowing ion events. About $50 \%$ of ion conics are found to be coincident with field-aligned electron beams. There is also a correlation in energy between the field-aligned electrons and ion conics. These show that the association is not a mere coincidence but rather that the field-aligned acceleration of electrons is related to the perpendicular energization of ions. The association rate of upflowing electrons is high on the nightside, while that of downflowing electrons is high on the dayside. The association rate of downflowing electrons is high at low altitudes, and the rates of the three types of electron beams become equivalent at high altitudes. Data indicate that the height of the electron acceleration region is lower on the nightside. It is suggested that the average height of the electron acceleration region is around the satellite apogee $(-10000 \mathrm{~km})$, and the average thickness of the region is about thousands $\mathrm{km}$.
\end{abstract}

Key words. Ionosphere-magnetosphere interactions . Particle acceleration - Auroral phenomena

\section{Introduction}

There have been several reports on field-aligned electron beams over the auroral region. Sharp et al. (1980) and Lin et al. (1982) reported counterstreaming electron

Correspondence to: W. Miyake beams using data from S3-3 and DE-1 satellites, respectively. Klumpar and Heikkila (1982) discovered field-aligned upflowing electrons at low altitude, using data from the ISIS-2 satellite. These electrons are aligned to within $10^{\circ}$ of the local magnetic field and have energies of several tens of $\mathrm{eV}$ to a few $\mathrm{keV}$.

The field-aligned electron beams are frequently spatially associated with upward-flowing ions with conical pitch angle distributions (i.e., ion conics) such as have been described in Sharp et al. (1977). Ion conics have cone-shaped intensity maxima centered on the magnetic field line direction in velocity space and are categorized into two types. Standard conics have an angular distribution with flux maxima at a nearly constant angle over an extended energy range (Sharp et al., 1977). The simple explanation for the generation is that they are the result of perpendicular acceleration of ions at low altitudes and the subsequent upward motion of the accelerated ions in the earth's mirror magnetic field conserving the first adiabatic invariant. Another type of ion conic is called an elevated (or bi-modal) conic (Klumpar et al., 1984). These have a pitch angle distribution at their high energy portion similar to standard conics, but the pitch angle distribution becomes field-aligned at their low energy portion. Various processes ranging from acceleration by a static electric field to energization by waves have been proposed as a cause of perpendicular acceleration of ions (see, for a review, Klumpar, 1986).

Although simultaneous observation of field-aligned electron beams and ion conics have been mentioned by many authors (Sharp et al., 1980; Klumpar and Heikkila, 1982; Lin et al., 1982; Burch et al. 1983; Kintner and Gorney, 1984; Gorney et al., 1985), only a few studies have been made on statistics of the correlation or the association. Collin et al. (1982) made a statistical study on the occurrence and characteristics of field-aligned electron beams based on S3-3 observation. They found that the latitude distribution of electron beams is related to that of the upflowing ions and that upflowing ions were coincident with electron beams in 
$30 \%$ of cases. Thelin and Lundin (1990) also made a statistical study of the distributions in MLT, Inv. lat., and altitude of field-aligned upflowing electrons and upflowing ions from Viking observations. They found a correlation between ion conics and electron beams in the distributions in magnetic local time, invariant latitude, and altitude, and suggested that ion conics and electron beams emanate from the same physical process.

Gorney et al. (1985) argued that the downward fieldaligned dc electric field accelerates the cold ionospheric electrons upward, resulting in the upward field-aligned electrons beams. Lin et al. (1982) also proposed a kind of dc potential island in the midst of the field line, in which the counterstreaming electrons are generated. Electron acceleration by an ac electric field was also proposed (Hultqvist et al., 1988; Boehm et al., 1995). Hultqvist $(1988,1991)$ studied possible electron acceleration along the field-line by low-frequency electric field fluctuations.

There are increasing suggestions on the close relation between the generation mechanisms of field-aligned electron beams and ion conics (see, for example, Hultqvist et al., 1988). After Collin et al. (1982) made a report on the association rate, no further study was made on the statistics of the association. In this paper we report statistical characteristics of the field-aligned electron beams associated with upflowing ion conics based on Exos-D (Akebono) observations. The purpose of our study here is to obtain more detailed relations between the field-aligned electrons and the ion conics through a statistical analysis.

\section{Data base}

The data for this study were acquired by the LEP instrument on the polar-orbiting Exos-D (Akebono) satellite with an initial apogee and perigee of 10482 and $272 \mathrm{~km}$, respectively (Oya and Tsuruda, 1990). The LEP instrument consists of two sets of $\mathrm{E} / \mathrm{Q}$ analysers and was designed to observe energy-pitch angle distributions of auroral electrons and ions. It has the energy range of 10 $\mathrm{eV}-16 \mathrm{keV}$ for electron measurement and $13 \mathrm{eV} / \mathrm{q}-20$ $\mathrm{keV} / \mathrm{q}$ for ion measurement, respectively. The pitch angle distribution of electrons and ions is measured by combining ten detectors with different incidence directions and satellite spin with a rate of about $7.5 \mathrm{rpm}$. The details of the instrument are given in Mukai et al. (1990).

The observation has been successfully carried out since the initial turn-on and a large data base for auroral electrons and ions has been built. The data used were obtained from April 1989 through April 1992. We used the same data set as used in the study of Miyake et al. (1996), in which all upflowing ion events are categorized into standard ion conics, elevated ion conics, and ion beams by means of an automated identification technique. The same automated identification process was applied for the identification of field-aligned electron beams.

In Fig. 1, upflowing ions and field-aligned electrons are seen in the energy-pitch angle diagrams. The left side of each panel shows the electrons and the right the ions. The intensity represents the count rate (e.g., the differential energy flux) on a logarithmic scale (see the right

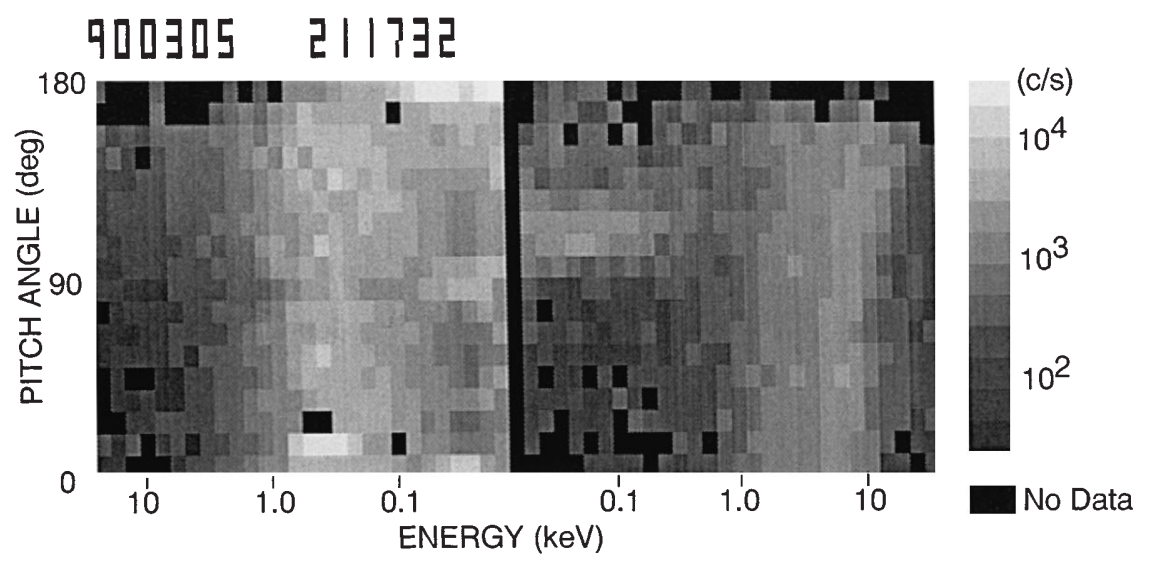

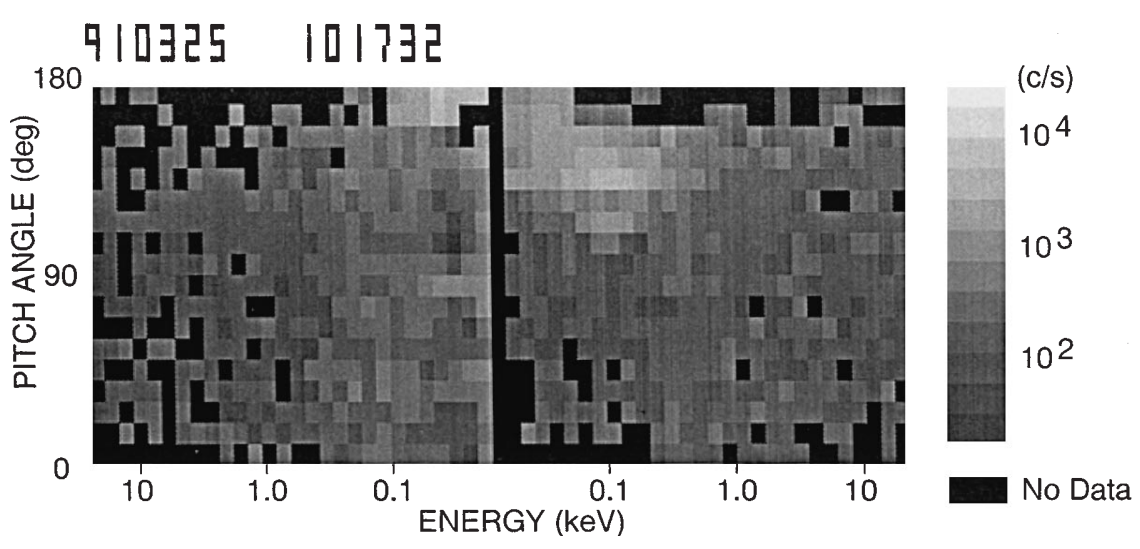

Fig. 1. An example of the simultaneous observation of the energy-pitch angle distribution of field-aligned electron beams and ion conics. The left side shows electrons and the right side ions. The intensity represents the count rate on a logarithmic scale as indicated on the right side. Each panel is made from integration of 16 -s observation. The low-energy electrons are narrowly collimated along the field line. The upper panel shows the counterstreaming electrons and the lower the upflowing electrons 
side). In the figure $180^{\circ}$ refers to upgoing particles and $0^{\circ}$ to downcoming particles. One panel is made from the integration of 16-s observation. All the data were displayed in this format of $29 \times 18$ energy-pitch angle bins and we made the identification of both the fieldaligned electron beams and upflowing ions automatically on the diagram.

In the upper panel, precipitating particles are found at the energy range of $100 \mathrm{eV}-1 \mathrm{keV}$ of electrons and of $1 \mathrm{keV}-10 \mathrm{keV}$ of ions. They have a wide pitch angle distribution. The electrons and ions with a narrow pitch angle distribution are seen in the low energy range. The energy-pitch angle diagram of ions shows ion conics with a pitch angle of $110^{\circ}-120^{\circ}$ up to $200 \mathrm{eV}$, while counterstreaming electrons appear along the field line (e.g., $0^{\circ}-10^{\circ}$ and $170^{\circ}-180^{\circ}$ bins) up to about $100 \mathrm{eV}$. In the lower panel, ion conics are also found in the diagram of ions and are associated with field-aligned upflowing electrons. The low-energy electrons with a pitch angle at $90^{\circ}$ in both the panels are believed to be the photoelectrons emitted from the satellite. They return to the satellite after a gyration around the magnetic field line.

The common condition for the field-aligned electron beams and all kinds of upflowing ions is that a significant anisotropic angular distribution must exist for at least three consecutive energy bands. The ridge (or the path) of the significant flux is traced in an energypitch angle diagram. The ridge must be constant in pitch angle or it gradually becomes perpendicular to the field line with increasing energy. Several ridge patterns usually satisfy the conditions. Among the possible ridge patterns, we select the ridge along which the integration of the count rate is largest. The categorization of upflowing ions is made by using the ridge pattern. See Miyake et al. (1996) and references therein for the details of the automated identification process.

\section{Basic properties of electron beams}

Figure 2 shows the angular distribution of the ridge at the maximum energy of field-aligned electrons with respect to the magnetic field line. The field-aligned electrons are categorized into three types as upflowing, downflowing, and counterstreaming cases by the flowing direction. The counterstreaming type is further subdivided into the upward component and downward component. All four kinds of electron beam are strongly field-aligned. There is no significant difference in the angular distribution among the four kinds. They, however, have a peak at $10^{\circ}-20^{\circ}$ angles from the field line. We believe that the distribution in Fig. 2 is affected by the lack of data along the field line direction. As seen in Fig. 1, an energy-pitch angle bin near the field line direction sometimes contains no available data in 16-s integration. This leads to a lower detection rate of electron beams with $0^{\circ}-10^{\circ}$ angles. The true angular distribution is believed to be more narrowly collimated along the field line. Collin et al. (1982) reported that the median half width at half maximum is $6^{\circ}$.

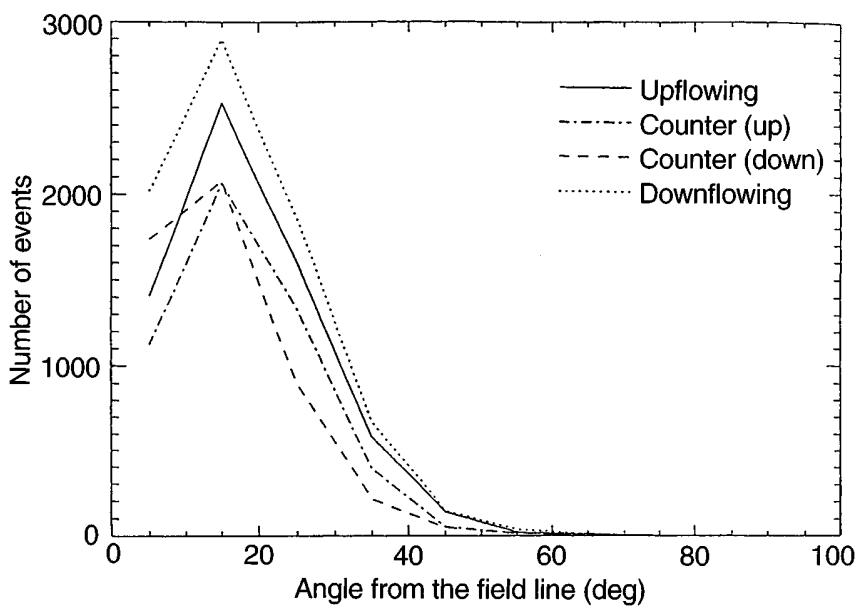

Fig. 2. Angular distribution of the electron beams associated with upflowing ion events. The electron beams are divided into the four types as indicated in the figure

The downflowing electron beams and the downward component of the counterstreaming electron beams have angles from the field line as narrowly collimated as the upflowing ones. This result indicates that the source region of these downcoming electrons is very close to the satellite. Otherwise the geomagnetic mirror force would widen the angular distribution of the downcoming electrons.

Figure 3 shows the distribution of the maximum energy of field-aligned electron beams associated with upflowing ion events. The electron beams are divided into the four types as in Fig. 2. Although some reports mentioned that the field-aligned electrons have energies of a few hundred $\mathrm{eV}$ up to the $\mathrm{keV}$ range (see, for example, Hultqvist et al., 1988), our observation shows that most of the field-aligned electrons have energies lower than $100 \mathrm{eV}$. Collin et al. (1982) reported that 70\% of electron beams were detected only in their lowest energy spectrometer with an energy range of $74-240 \mathrm{eV}$.

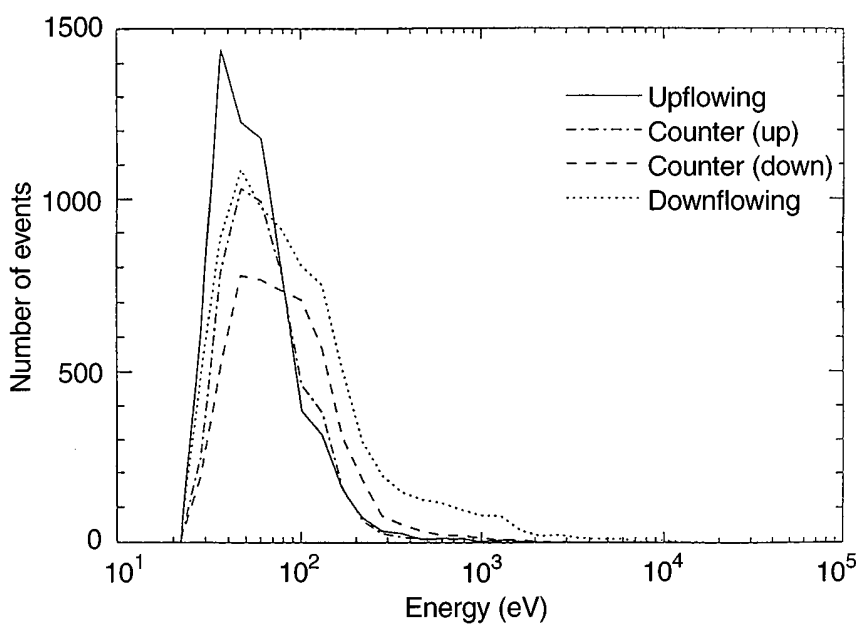

Fig. 3. Energy distribution of field-aligned electron beams associated with upflowing ion events. The four curves represent the four types of electron beam as shown in the figure 

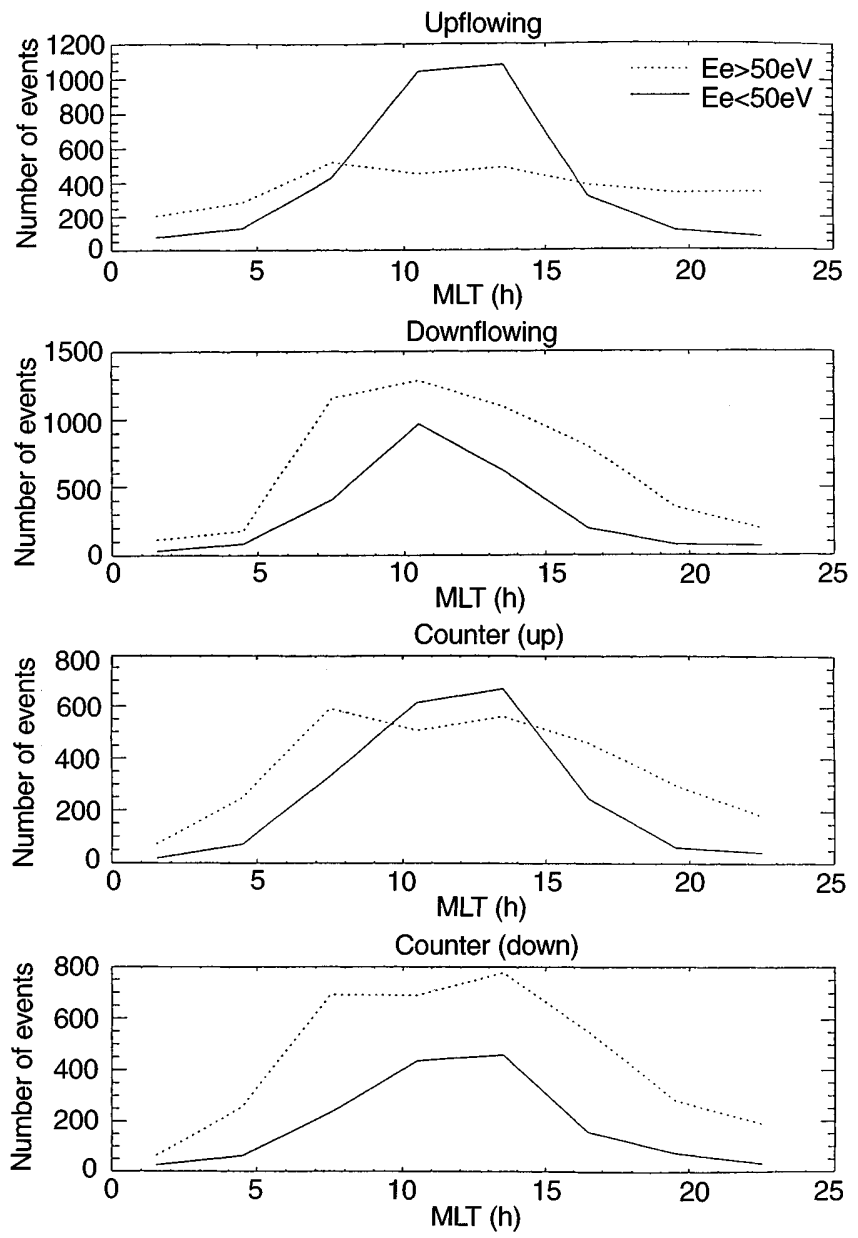

Fig. 4. MLT distribution of the four types of field-aligned electron beam. The electron beams are divided into two by their energy $(E e)$

There is only a slight difference in the distribution among the four types. The downflowing electrons generally have the highest energies among the four. Similarly, the downflowing component of the counterstreaming electrons tends to have a higher energy. The energy of the upflowing electrons is lowest.

The MLT distribution of field-aligned electron beams is presented in Fig. 4. The field-aligned electron beams are divided into the four types again and are also subdivided by their maximum energy. All the four types of field-aligned electron beams show a dayside concentration, which is believed to reflect the dayside peak of the occurrence of ion conics (see, for example, Miyake et al., 1996). As shown later in Table 1, most of the fieldaligned electrons presented in this study are associated with ion conics.

Table 1. Association rates of the three types of field-aligned electron beams with the three types of upflowing ions: ion beams, elevated conics, and standard conics

\begin{tabular}{llll}
\hline & Ion beams & Elevated conics & Standard conics (\%) \\
\hline Up only & 7 & 14 & 15 \\
Counter & 4 & 14 & 12 \\
Down only & 7 & 20 & 19 \\
\hline
\end{tabular}

We do not present the occurrence frequencies here but the mere number of observed events, so that the comparison between different MLTs is not meaningful. The figure rather shows a dependence of the electron energy on MLT. It is clear again in Fig. 4 that the downflowing electrons have higher energies than the upflowing electrons. This dependence of energy on the type of field-aligned electron beams mostly comes from the events on the dayside, where most of the events exist. On the nightside the electron beams with energies larger than $50 \mathrm{eV}$ are dominant for all the four types. The energy of all types of field-aligned electron beam is generally low on the dayside and high on the nightside.

\section{Association rate}

The association rates of the field-aligned electron beams for the three kinds of UFI; ion beams, elevated conics, and standard conics, are summarized in Table 1. In the analysis, we first identify the upflowing ion events and categorize them into the three kinds. Then, we look through the electron data at the time when those ion events are identified. The association rate here is defined as the ratio of the number of field-aligned electron events observed simultaneously with a type of upflowing ion event to the number of the type of upflowing ion event. Both types of ion conic have higher association rates than ion beams. This result is coincident with that of Collin et al. (1982), in which the association rates are presented for ion beams and ion conics based on S3-3 observations. The higher association with ion conics than with ion beams agrees with closely related processes being involved in the field-aligned acceleration of electrons and the perpendicular energization of ions, which several previous reports on simultaneous observation of field-aligned electron beams and ion conics have suggested. Table 1 shows that field-aligned electron beams are observed simultaneously with about $50 \%$ of ion conics. Taking into account the low rate of detection owing to the lack of data along the field line, the high association rate is notable. Because of the low association rate, which also means small number of events, with ion beams, we hereafter deal only with the electron beams associated with ion conics for the analysis.

Another similarity to the results of Collin et al. (1982) is that the association rate of the downflowing electron beams is highest among all the types of electron beam. This result suggests, however, a complicated relation between the field-aligned acceleration of electrons and the perpendicular energization of ions rather than a straightforward mechanism. Some previous studies (Gorney et al., 1985; Hultqvist et al., 1988; Boehm et al., 1995) focus on the simultaneous observation of upflowing electron beams and ion conics. They discuss possible mechanisms for accelerating both the electrons and ions from the same region. Our observation suggests the separation of the source regions of electron beams and ion conics along the field line.

One may consider that the association of field-aligned electron beams with ion conics is just a coincidence and 


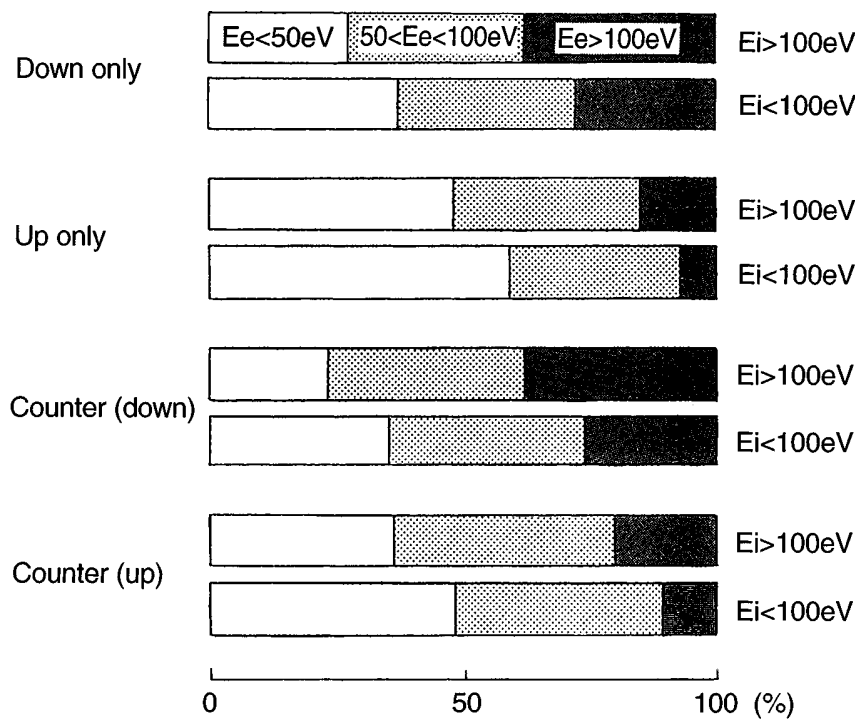

Fig. 5. Relationship of energy of ion conics $(E i)$ with that of the associated field-aligned electron beams $(E e)$

that there is no physical relation in their source mechanism. One of the possibilities is that, since it takes time for ions to flow up, a rapid temporal variation during the magnetically disturbed period may locate field-aligned electron beams on the same field line as ion conics. We examined a dependence of the association rate on the $\mathrm{Kp}$ index. The numbers of events of ion conics and field-aligned electrons are both increased with the Kp index. Their increases, however, are almost equivalent, and it results in no dependence of the association rate on $\mathrm{Kp}$ index.

We also examine the relationship between the energy spectra of the electron beams and ion conics. The result is shown in Fig. 5. The electron beams are divided into three energy ranges and ion conics into two energy ranges. $E e$ represents the maximum energy of electron beams and $E i$ that of ion conics. For all the types of field-aligned electrons, the ion conics with higher energies $(E i>100 \mathrm{eV})$ are associated with the electron beams with higher energies. No variation of the association rate with geomagnetic activity and the correlation in energy between the ion conics and the associated electron beams suggests that the simultaneous occurrence on the same field line is not just a coincidence but that their source mechanisms have some physical link.

Figure 6 shows the MLT dependence of the association rates with ion conics. The three curves correspond to the three types of field-aligned electron beam as indicated in the figure. Although the curves have large scatter, a difference is clear between the dayside and nightside. The association of the downflowing electron beams is dominant on the dayside, while that of the upflowing electron beams dominates on the nightside. Another feature in Fig. 6 is that there is a slight dawndusk asymmetry. The association rate for all the three types tends to be higher on the dawn side.

Figure 7 shows the altitude variation of the association rates with ion conics. The MLT dependence is

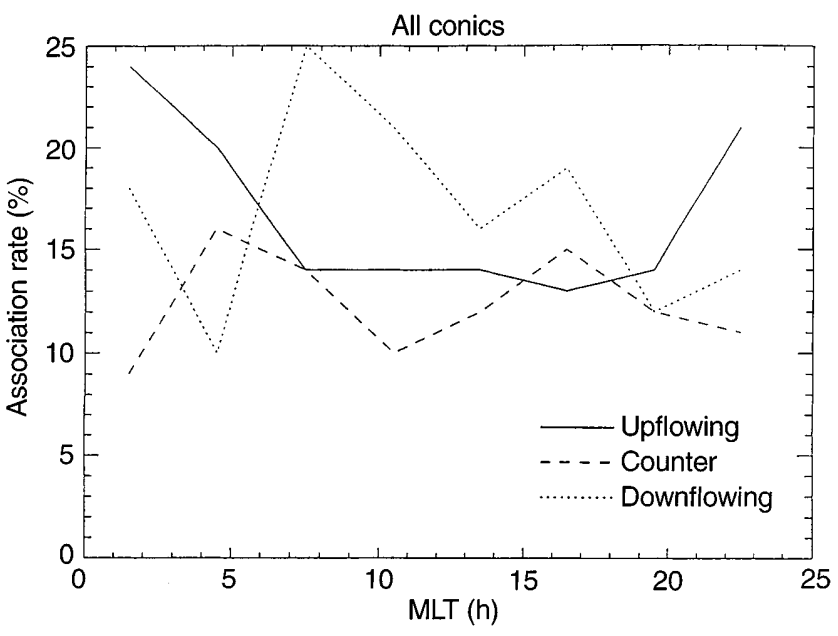

Fig. 6. MLT dependence of the association rate of electron beams with ion conics. The three curves in the figure correspond to the three types of the electron beam
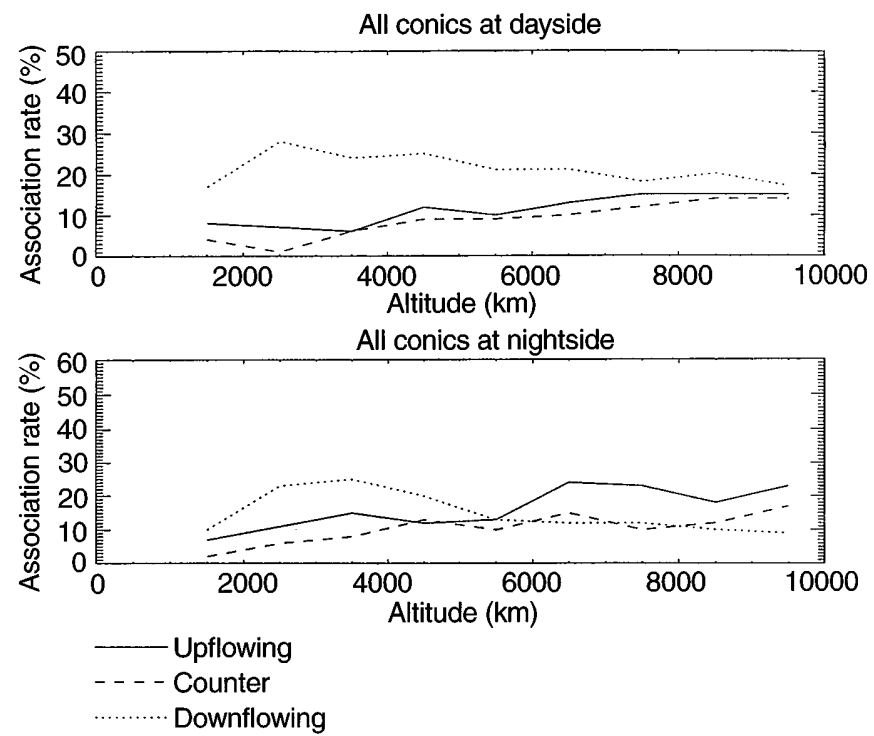

Fig. 7. Altitude variation of the association rate of the three types of field-aligned electron beams with ion conics

different between the upflowing and downflowing electron beams as in Fig. 6, so that we examine the altitude variation by dividing the events into the dayside events (upper panel) and the nightside events (lower panel). The types of field-aligned electron beam are indicated in the same way as in Fig. 6. The association rate of both the upflowing and counterstreaming electron beams is increased with increasing altitude, while the rate of the downflowing electron beams is high at low altitude and is decreased with increasing altitude. The difference in the rate between the upflowing and downflowing electron beams becomes smaller at high altitude on the dayside. On the nightside, the rate of the downflowing electrons becomes smaller than that of the others at high altitude. Therefore, it is concluded that the higher association rate of the downflowing electrons shown in Table 1 is due to the contribution of the dominant 
dayside events (see Fig. 4) to the statistics, and the difference is not so large if we take only the high-altitude events in the analysis.

We present the association rates but not the occurrence frequencies of field-aligned electron beams here. By multiplying the occurrence frequencies of ion conics (see Fig. 2 of Miyake et al., 1996), we obtain the occurrence of downflowing electron beams which is increased with increasing altitude. The purpose of this study, however, is to investigate the relationship between the field-aligned electron beams and the upflowing ion events on the same field line. Therefore, we do not treat the occurrence frequencies of the field-aligned electron beams in this paper.

\section{Discussion}

The angular distribution of the field-aligned electron beams in Fig. 2 shows only a slight difference among the four types of electron beam. The four types of fieldaligned electron beam also have a similar energy distribution as in Fig. 3. Furthermore, ion conics have a good correlation with field-aligned electron beams, but ion beams do not. Therefore, we have no reason to distinguish the downcoming electron beams from the upgoing electron beams regarding the acceleration mechanism. The same source mechanism is suggested to be responsible for the acceleration of all the types of field-aligned electron beams, which means that an ac acceleration mechanism is favored.

Klumpar et al. (1988) observed counterstreaming electrons at the geomagnetic equator and suggested that the electrons are emanated from the auroral acceleration region of both hemispheres. We cannot rule out the possibility that a fraction of the downcoming components of the counterstreaming electrons observed by Exos-D come from the other hemisphere. A large fraction of all the types of electron beam are, however, associated with ion conics and are observed at the cusp/ cleft regions (Fig. 4), where not all the field lines are expected to be closed.

It may also be possible that the downcoming components of the counterstreaming electrons are explained by reflection of the upflowing electron beams at a dc potential barrier above the satellite. The occurrence of ion beams is, however, higher on the evening side [see, for example, Miyake et al. (1996) and references therein], which is believed to be due to the higher occurrence of dc parallel acceleration on the evening side. The MLT distribution of the counterstreaming and downflowing electrons (Fig. 6) has no significant displacement toward the afternoon sector compared with that of the upflowing electron beams. As shown in Table 1, the counterstreaming electrons have a low association rate with ion beams. The ion beams indicate the parallel acceleration below, but not above, the satellite, but we can assume the potential drop above the satellite when we detect ion beams. Since the satellite altitude is not so high that it is seldom located completely above the parallel dc acceleration region, we almost always see the magnetospheric electrons accelerated downward above the satellite with the ion beams accelerated below the satellite. Therefore, it seems that the reflection of the upflowing electron beams at the potential barrier above the satellite can account for only a small fraction of the counterstreaming electron beams.

We obtained an association rate of about $50 \%$, which seems to agree with the association rate found by Collin et al. (1982) from S3-3 observation. The true rate may possibly be higher since the observation in the direction of the field line is sometimes incomplete, which leads to a lower detection rate of the field-aligned electron beams. This high association rate suggests that the association is not a mere coincidence but rather that both the acceleration mechanisms responsible for electrons and ions are related to each other. It is also supported by no dependence of the association rate on $\mathrm{Kp}$ index.

Gorney et al. (1985) argued that a de downward electric field is the probable cause of the acceleration of upward field-aligned electron beams, so that the ion conics can be trapped in the downward electric field, which results in the acceleration of ions up to high energies owing to the increase in the residence time in the perpendicular acceleration region. Hulqvist et al. (1988), on the other hand, proposed that a lowfrequency ac process is responsible for both the fieldaligned acceleration of electrons and the perpendicular energization of ions. In either case, we expect a correlation between the acceleration of electrons and ions. Our results demonstrate the correlation of the energy of ion conics with the energy of field-aligned electron beams.

The model of fluctuating electric field can also provide an explanation of both the counterstreaming and downflowing electrons, though Hultqvist's original model does not contain the downflowing electrons. The satellite observes the downflowing electron beams when it is located below the acceleration region and it observes the counterstreaming electron beams when it is in the midst of the acceleration region. The electrons are not simultaneously counterstreaming in the acceleration region but are rather switched between upflowing and downflowing following the phase of the electric field fluctuation. The integration time of the particle measurement is longer than the characteristic time-scale of the fluctuation, which leads to the detection of fieldaligned 'counterstreaming' electron beams.

The observation shows that higher correlation of ion conics with downflowing electron beams than with upflowing and counterstreaming electron beams, especially at low altitude. This means that the perpendicular energization region of ions is below and the parallel acceleration region of electrons is above the satellite. The parallel acceleration region of electrons and perpendicular energization region of ions are often separated in altitude on the same field line.

The difference in altitude between the ion energization and the electron acceleration region is not so large, since the downflowing electron beams are collimated along the field line as narrowly as the upflowing electron 
beams are (Fig. 2). This is also supported by the altitude variation of the association rate in Fig. 7. The association rate of downflowing electron beams is higher at lower altitude, while it becomes equivalent to both the rates of the upflowing and counterstreaming electron beams at higher altitude. This suggests that the average height of the source region of the electron beams is just above the satellite apogee on the dayside and is even just below it on the nightside.

The acceleration region of field-aligned electron beams can be lower on the nightside than on the dayside. This dependence is similar to that of ion conics. The perpendicular energization region of ions is located above a few thousand $\mathrm{km}$ on the dayside cusp/cleft region (Whalen et al., 1991; Knudsen et al.,1994), while energetic ion conics are observed by sounding rockets at several hundreds $\mathrm{km}$ of altitude over the nightside ionosphere (Yau et al., 1983).

Hultqvist et al. (1991) investigated the dependence of field-aligned electron beam generation on plasma density and found that low plasma density favors the generation of field-aligned electron beams and that a high density hinders it. It is probable that lower plasma density tends to decrease the average height of the acceleration region of field-aligned electron beams at nightside. This preference of low plasma density may also account for the fact that the energy of the downcoming electron beams is higher than that of upgoing electrons (Fig. 3) and the fact that the energy of all the types of field-aligned electron beams is higher at nightside than at dayside.

If the counterstreaming case means that the satellite is located in the midst of the electron acceleration region, we can estimate the thickness of the acceleration region along the field line by comparing the association rate among the three types of field-aligned electron. The rate of counterstreaming electrons is almost equivalent to that of upflowing electron beams. This result suggests that the thickness of the acceleration region is about half of the altitude, i.e., a few to five thousand $\mathrm{km}$. If the thickness is smaller, then the satellite would be in the midst of an acceleration region in rare occasions and the rate of the counterstreaming case would be lower. If the thickness is larger, then the upflowing case would be seldom observed and the association rate of the counterstreaming electron beams would be much higher than that of the upflowing case. Hultqvist (1991) also obtained 1000-10000 km for the height extension of the acceleration region from a model calculation of electron motions within slow, large-amplitude fluctuations of electric field.

Most of our observation results are well explained by the electron acceleration owing to the parallel ac electric field. They are also supported by the high association rates and the correlation of energy of both the electrons and ions. The difference from the previous work (Hultqvist, 1988) we must note is that the parallel acceleration region of electrons tends to be located above the perpendicular energization region of ions rather than being emanated from the same source region.
Even if the same process is responsible for both the parallel acceleration of electrons and the perpendicular energization of ions, the component of the electric field which acts on electrons is different from that acting on ions. The fluctuating (or wave) electric field has a primary component perpendicular to the magnetic field line, which is believed to cause the perpendicular energization of ions. A small fraction of the electric field is directed parallel to the magnetic field line. This parallel component accelerates electrons along the field line. Therefore, in the case where the fluctuating (or wave) electric field is extended along the field line, the field-aligned acceleration region of electrons is not necessarily coincident with the perpendicular energization region of ions. They can sometimes be close to each other, or may even occupy the same region on the field line. The statistics just tell us that the electron acceleration region tends to be located a few to several thousand $\mathrm{km}$ higher than the ion energization region.

The possibility is not ruled out that the electron acceleration and ion energization are not due to the same fluctuating or wave electric field but due to two independent modes of waves. Their energy sources and/ or the excitation mechanisms are closely related, so that we may find the high association rate of the field-aligned electrons with ion conics and the correlation in energy between the electron beams and ion conics.

The electrons must be supplied from the magnetosphere or the ionosphere into the acceleration region. The source of electrons determines the contribution of field-aligned electron beams to the direction of the fieldaligned dc current there, since the ac acceleration region emits the electrons in both directions and makes no contribution to the dc current. The narrow angular distribution and low energy of the electron beams favor the ionospheric source. It is interesting to note that the higher association rate on the dawn side (Fig. 6) may be attributed to the downward current in Region 1 on the dawn side, though we must take into account the precipitation of magnetospheric electrons to determine the net current.

In summary, since all the types of field-aligned electron beams have a similar energy and angular feature and similar high association rates with upflowing ion events, it seems that all the types are generated through an identical acceleration process which has a relation to the perpendicular energization of ions. The MLT dependence and the altitude variation of the association rates suggest that the parallel electron acceleration favors a low density region. The parallel acceleration region of electrons tends to be located above the perpendicular energization of ions on the same field line. The average altitude of the electron acceleration region is estimated to be just above the satellite apogee $(-10000 \mathrm{~km})$ on the dayside and below it on the nightside.

Acknowledgements. We thank all the members of the Exos-D project team, especially K. Tsuruda and $\mathrm{H}$. Oya, for their extensive support. We are grateful to A. W. Yau for valuable discussion and useful comments on this work. 
Topical Editor K.-H. Glassmeier thanks J. L. Burch and B. Hultqvist for their help in evaluating this paper.

\section{References}

Boehm, M. H., J. Clemmons, J.-E. Wahlund, A. Eriksson, L. Eliasson, L. Blomberg, P. Kintner, and H. Hofner, Observations of an upward-directed electron beam with the perpendicular temperature of the cold ionosphere, Geophys. Res. Lett., 22, 2103, 1995.

Burch, J. L., P. H. Reiff, and M. Sugiura, Upward electron beams measured by DE-1: a primary source of dayside region-1 Birkeland currents, Geophys. Res. Lett., 10, 753, 1983.

Collin, H. L., R. D. Sharp, and E. G. Shelley, The occurrence and characteristics of electron beams over the polar regions, $J$. Geophys. Res., 87, 7504, 1982.

Gorney, D. J., Y. T. Chiu, and D. R. Croley Jr., Trapping of ion conics by downward parallel electric fields, J. Geophys. Res., 90, 4205, 1985.

Hultqvist, B., On the acceleration of electrons and positive ions in the same direction along magnetic field lines by parallel electric fields, J. Geophys. Res., 93, 9777, 1988.

Hultqvist, B., On the motion of electrons in the slow electric field fluctuations observed by Viking, J. Geophys. Res., 96, 19513, 1991.

Hultqvist, B., R. Lundin, K. Stasiewics, L. Block, P.-A. Lindqvist, G. Gustafsson, H. Koskinen, A. Bahnsen, T. A. Potemra, and L. J. Zanetti, Simultaneous observation of upward moving fieldaligned energetic electrons and ions on auroral zone field lines, J. Geophys. Res., 93, 9765, 1988.

Hultqvist, B., H. Vo, R. Lundin, B. Aparicio, P.-A. Lindqvist, G. Gustafsson, and B. Holback, On the upward acceleration of electrons and ions by low-frequency electric field fluctuations observed by Viking, J. Geophys. Res., 96, 11609, 1991.

Kintner, P. M., and D. J. Gorney, A search for the plasma processes associated with perpendicular ion heating, J. Geophys. Res., 89, 937, 1984

Klumpar, D. M., A digest and comprehensive bibliography on transverse auroral ion acceleration, in Ion acceleration in the magnetosphere and ionosphere, Geophys. Monogr. Ser., Vol. 38, Ed. T. Chang, AGU, Washington DC, pp. 389-398, 1986.
Klumpar, D. M., and W. J. Heikkila, Electrons in the ionospheric source cone: evidence for runaway electrons as carrier of downward Birkeland currents, Geophys. Res. Lett., 9, 873, 1982.

Klumpar, D. M., W. K. Peterson, and E. G. Shelley, Direct evidence for two-stage (bimodal) acceleration of ionospheric ions, $J$. Geophys., Res., 89, 10779, 1984.

Klumpar, D. M., J. M. Quinn, and E. G. Shelley, Counterstreaming electrons at the geomagnetic equator near $9 \mathrm{Re}$, Geophys. Res. Lett., 15, 1295, 1988.

Knudsen, D. J., B. A. Whalen, T. Abe, and A. W. Yau, Temporal evolution and spatial dispersion of ion conics: evidence for a polar cusp heating wall, in Solar system plasmas in space and time, Geophys. Monogr. Ser., Vol. 84, Eds. J. L. Burch and J. H. Waite Jr., AGU, Washington DC, pp. 163-169, 1994.

Lin, C. S., J. L. Burch, J. D. Winningham, and J. D. Menietti, DE 1 observations of counter-streaming electrons at high altitudes, Geophys. Res. Lett., 9, 925, 1982.

Miyake, W., T. Mukai, and N. Kaya, On the origins of the upward shift of elevated(bi-modal) ion conics in velocity space, $J$. Geophys. Res., 101, 26961, 1996.

Mukai,T., et al., Low-energy charged particle observations in the 'auroral' magnetosphere: first results from Akebono (Exos-D) satellite, J. Geomagn. Geoelectr., 42, 479, 1990.

Oya, H., and K. Tsuruda, Introduction to the Akebono (Exos-D) satellite observations, J. Geomagn. Geoelectr., 42, 367, 1990.

Sharp, R. D., R. G. Johnson, and E. G. Shelley, Observations of an ionospheric acceleration mechanism producing energetic $(\mathrm{keV})$ ions primarily normal to the geomagnetic direction, J. Geophys. Res., 82, 3324, 1977.

Sharp, R. D., E. G. Shelley, R. G. Johnson, and A. G. Ghielmetti, Counterstreaming electron beams at altitudes of -1 Re over the auroral zone, J. Geophys. Res., 85, 92, 1980.

Thelin, B., and R. Lundin, Upflowing ionospheric ions and electrons in the Cusp-Cleft region, J. Geomagn. Geoelectr., 42, $753,1990$.

Whalen, B. A., S. Watanabe, and A. W. Yau, Observations in the transverse ion energization region, Geophys. Res. Lett., 18, 725, 1991.

Yau, A. W., B. A. Whalen, A. G. McNamara, P. J. Kellog, and W. Bernstein, Particle and wave observations of low-altitude ionospheric ion acceleration events, J. Geophys. Res., 88, 341, 1983. 